\title{
MicroRNA Expression as a Predictive Marker for Gemcitabine Response after Surgical Resection of Pancreatic Cancer
}

\author{
Kenoki Ohuchida, $\mathrm{PhD}^{1,2}$, Kazuhiro Mizumoto, $\mathrm{PhD}^{1,3}$, Tadashi Kayashima, $\mathrm{MD}^{1}$, Hayato Fujita, $\mathrm{PhD}^{1}$, \\ Taiki Moriyama, $\mathbf{P h D}^{1}$, Takao Ohtsuka, $\mathrm{PhD}^{1}$, Junji Ueda, $\mathbf{P h D}^{1}$, Eishi Nagai, $\mathbf{P h D}^{1}$, Makoto Hashizume, $\mathbf{P h D}^{2}$, \\ and Masao Tanaka, $\mathbf{P h D}^{1}$ \\ ${ }^{1}$ Department of Surgery and Oncology, Graduate School of Medical Sciences, Kyushu University, Fukuoka, Japan; \\ ${ }^{2}$ Department of Advanced Medical Initiatives, Graduate School of Medical Sciences, Kyushu University, Fukuoka, Japan; \\ ${ }^{3}$ Kyushu University Hospital Cancer Center, Fukuoka, Japan
}

\begin{abstract}
Background. To improve the prognosis of patients after resection of pancreatic cancer, the most appropriate and efficient treatment should be provided to specific subsets of patients. Our aim was to identify promising microRNAs as markers to predict responses to gemcitabine in patients with resected pancreatic cancer.

Methods. Two gemcitabine-resistant pancreatic cancer cell lines were established, and global microRNA expression analyses was performed by quantitative reverse transcriptionpolymerase chain reaction (qRT-PCR). Eleven miRNAs were selected as putative predictive markers and analyzed by means of macrodissected formalin-fixed, paraffin-embedded samples obtained from 90 patients with or without gemcitabine treatment after resection of pancreatic cancer.

Results. We identified 24 microRNAs whose expression was altered in gemcitabine-resistant cells. qRT-PCR analyses showed that patients with high miR-142-5p and miR204 expression had significantly longer survival times than those with low miR-142-5p $(P=0.0077)$ and miR-204 $(P=0.0054)$ expression in the gemcitabine-treated group. This was not seen in the nontreated group. Multivariate analyses showed that miR-142-5p expression was an independent prognostic marker only in patients treated with gemcitabine $(P=0.034)$.
\end{abstract}

(C) The Author(s) 2011. This article is published with open access at Springerlink.com

First Received: 18 November 2010;

Published Online: 23 February 2011

K. Ohuchida, $\mathrm{PhD}$

e-mail: kenoki@surg1.med.kyushu-u.ac.jp

K. Mizumoto, $\mathrm{PhD}$

e-mail: mizumoto@med.kyushu-u.ac.jp
Conclusions. miR-142-5p is a promising predictive marker for gemcitabine response in patients with resected pancreatic cancer.

Pancreatic cancer is the fourth most common cause of tumor-related death in the industrialized world. ${ }^{1,2}$ Only $10-20 \%$ of pancreatic cancer patients are candidates for surgery at the time of presentation, and fewer than $20 \%$ of patients who undergo curative resection are alive after 5 years. ${ }^{3,4}$ A few recent reports have described the successful use of adjuvant chemotherapies such as 5-fluorouracil and gemcitabine. ${ }^{5,6}$ However, not all patients benefit from such adjuvant chemotherapy, and we cannot predict which patients will benefit most from this treatment. Therefore, to improve the prognosis of patients with resected pancreatic cancer, we need to identify specific markers that can predict responses to adjuvant therapy. Such a personalized therapy, based on the predictive markers, may provide the most appropriate and efficient treatment for a specific subset of patients.

Gemcitabine is a deoxycytidine analog with antitumor activity that bears a resemblance, both structurally and metabolically, to arabinosyl cytosine. ${ }^{7}$ Gemcitabine is widely accepted as the first-line treatment for patients with advanced or resected pancreatic cancer. ${ }^{6,8}$ However, recent reports showed that the complete plus partial response rate and the disease control rate in advanced pancreatic cancer are $8.0-13.5 \%$ and $49.2-62.1 \%$, respectively, even with combination-treatment arms. ${ }^{9,10}$ The data suggest that approximately half of patients with resected pancreatic cancer do not benefit from gemcitabine-based combination therapies. Therefore, predictive markers are needed to select those patients who may benefit most from gemcitabine-based therapy. So far, research into the mechanism of 
resistance to gemcitabine has led to identification of several candidate predictive markers. These include genes related to gemcitabine metabolism and transport, such as deoxycytidine kinase, ribonucleotide reductase, and human equilibrative nucleoside transporter- $1 .{ }^{11,12}$ However, the usefulness of such markers in a clinical setting remains unclear because of difficulties in evaluating their protein or mRNA levels. Even when well-established methods are used, immunohistochemical evaluation and its interpretation are different between laboratories. ${ }^{13}$ Also, accurate quantitative analyses of mRNA from clinical samples are often difficult as a result of degradation. Therefore, we need more reliable methods-based biomarkers to predict responses to gemcitabine.

MicroRNAs (miRNAs) are small noncoding RNA gene products of approximately $22 \mathrm{nt}$ that are found in a variety of organisms. They play key roles in regulating the translation and degradation of mRNAs through base pairing to partially complementary sites, predominantly in the $3^{\prime}$-untranslated regions of mRNAs. ${ }^{14,15}$ Because microRNAs are well preserved, even in formalin-fixed, paraffin-embedded (FFPE) samples, the quantitative analysis of miRNA in many types of clinical sample is accurate and reliable. ${ }^{16,17}$ Therefore, miRNAs are promising molecular markers.

There is increasing evidence that miRNAs are mutated or differentially expressed in many types of cancers, and thus are also potential diagnostic markers. ${ }^{18-20}$ The expression levels of several miRNAs, such as miR-21, miR-196a-2, miR-155, and miR-210 in pancreatic cancer, are known to correlate with a poor prognosis. ${ }^{19,21,22}$ Thus, there is a possibility that specific miRNAs that can be used as predictive markers for a gemcitabine response exist.

Here, to identify predictive markers, we established two gemcitabine-resistant pancreatic cancer cell lines and performed global miRNA expression analyses. We then further investigated 11 selected miRNAs as potential predictive markers using macrodissected FFPE samples derived from 90 patients with resected pancreatic cancer.

\section{MATERIALS AND METHODS}

\section{Cell Lines and Establishment of Gemcitabine-Resistant Cells}

Two human pancreatic cancer cell lines, SUIT-2 and CAPAN-1 (the gift of Dr. H. Iguchi, National Shikoku Cancer Center, Matsuyama, Japan), were used in this study. Gemcitabine-resistant cells were generated by exposing these cell lines to gradually increasing concentrations of gemcitabine. The initial concentration of gemcitabine was $1 \mathrm{nM}$, which did not seem to affect the proliferation of either the SUIT-2 or CAPAN-1 cells. When the cells had adapted to the drug, the concentration of gemcitabine was gradually increased by $10-100 \mathrm{nM}$ per week to a final concentration of $200 \mathrm{nM}$ (SUIT-2) and $1 \mu \mathrm{M}$ (CAPAN-1). Gemcitabine was dissolved in phosphate-buffered saline and added to the cell culture media.

\section{Propidium Iodide Assay}

Cell proliferation was evaluated by measuring the fluorescence intensity of propidium iodide as described previously. ${ }^{23}$ The fluorescence intensity corresponding to the total cells was measured with a CytoFluor multiwell plate reader (PerSeptive Biosystems, Framingham, MA, USA). The results were converted to percentage survival rates by comparing treated cells with untreated cells.

\section{miRNA Expression Profiling}

Expression profiles for the 365 mature miRNAs were obtained by quantitative polymerase chain reaction (qPCR) using the TaqMan Array Human MiRNA (TLDA) or TaqMan MiRNA Assays (Applied Biosystems; ABI) on an ABI Prism 7900HT according to the manufacturer's instructions. Mature miRNAs were normalized to RNU44 and are expressed as fold changes relative to controls.

\section{Patients and Pancreatic Tissues}

From 1992 through 2008, a total of 104 patients underwent pancreatic resection for pancreatic cancer at the Department of Surgery and Oncology, Kyushu University Hospital (Fukuoka, Japan). Survival was measured from the time of pancreatic resection, and death was the end point. The follow-up data for 103 cases were available. Thirteen of the 103 patients were excluded from the present study because they had received combination therapy with gemcitabine and other chemotherapeutic agents such as S1(tegafur/gimestat/potassium oxonate).

Our final study series consisted of 90 patients with resected pancreatic cancer with available follow-up data. The patients (57 men and 32 women) had a median age of 65 years (range, 36-86 years). The median observation time for overall survival was 14.7 months, ranging 0.5-108 months. Sixty-three patients died during followup; the other patients were alive and censored.

All resected specimens were fixed in formalin and embedded in paraffin for pathological diagnosis. All tissues adjacent to the specimens were evaluated histologically according to the criteria of the World Health Organization. ${ }^{24}$ Diagnoses were confirmed independently by two pathologists with regard to the pathological features of all cases. Tumor stage was assessed according to the International Union Against Cancer classification. ${ }^{25}$ Clinicopathological 
characteristics of the tumors are shown in Table 1 . The study was approved by the Ethics Committee of Kyushu University and was conducted according to the Ethical Guidelines for Human Genome/Gene Research enacted by the Japanese government and the Helsinki Declaration.

\section{Macrodissection}

After a review of representative hematoxylin and eosinstained slides, 4-7 sections ( $5 \mu \mathrm{m}$ thick) were cut from FFPE blocks for macrodissection. Adjacent normal tissues, including normal acinar tissues and adipose tissues, in the sections were removed macroscopically with a scalpel. Only the cancerous parts were used for the isolation of miRNA.

TABLE 1 Correlation between median OS and clinical and pathological factors in patients with resected pancreatic cancer

\begin{tabular}{|c|c|c|c|}
\hline Factor & $n$ & OS $(95 \% \mathrm{CI})$ & $P$ value \\
\hline Age & & & 0.675 \\
\hline$\geq 65$ years & 47 & $19(13.07-27)$ & \\
\hline$<64$ years & 42 & $19(12-26)$ & \\
\hline Sex & & & 0.7544 \\
\hline Male & 57 & $23(13.07-30.17)$ & \\
\hline Female & 32 & $14(10-24.2)$ & \\
\hline pT category & & & 0.0019 \\
\hline $\mathrm{pTl} / \mathrm{pT} 2 / \mathrm{pT} 3$ & 55 & $23(16.30-43)$ & \\
\hline $\mathrm{p} 4$ & 33 & $12(9.43-19)$ & \\
\hline pN category & & & 0.0070 \\
\hline pNO & 26 & $43(16.93-)$ & \\
\hline $\mathrm{pN} 1$ & 62 & $13.27(11.6-22)$ & \\
\hline Histological grade & & & 0.0804 \\
\hline G1 & 19 & $31(9-)$ & \\
\hline $\mathrm{G} 2$ & 33 & $23(14-30.16)$ & \\
\hline G3 & 35 & $12(10-23)$ & \\
\hline Residual tumor & & & $<0.0001$ \\
\hline $\mathrm{R} 0$ & 53 & $26(19-45)$ & \\
\hline $\mathrm{R} 1$ & 34 & $12(9-13.73)$ & \\
\hline Vessel invasion & & & 0.0204 \\
\hline Positive & 57 & $13.73(12-23)$ & \\
\hline Negative & 31 & $27(15-)$ & \\
\hline Lymphatic invasion & & & 0.3004 \\
\hline Positive & 76 & $15(12-24.2)$ & \\
\hline Negative & 18 & $27(14.53-)$ & \\
\hline Neural invasion & & & 0.6849 \\
\hline Positive & 73 & $16.93(13.07-26)$ & \\
\hline Negative & 15 & $23(4.7-)$ & \\
\hline Adjuvant therapy & & & 0.0382 \\
\hline Yes & 59 & $23(14.53-30.17)$ & \\
\hline No & 30 & $12(7.7-24.2)$ & \\
\hline
\end{tabular}

$O S$ overall survival, $C I$ confidence interval

\section{Isolation of miRNA}

miRNA was extracted from the macrodissected FFPE samples with the RNeasy FFPE kit (Qiagen, Tokyo, Japan) using a method modified from the manufacturer's instructions. Briefly, macrodissected FFPE sections were deparaffinized with xylene, washed with ethanol, and dried. Lysis buffer and proteinase $\mathrm{K}$ were added to the dried sections. The sections were incubated and Binding buffer was then added to the lysate and transferred to a gDNA Eliminator spin column (Qiagen) to remove genomic DNA. After eliminating DNA, $100 \%$ ethanol was added to the flow-through. After mixing, the samples were transferred to an RNeasy MinElute column (Qiagen), which binds total RNA. After washing, the purified RNA was eluted with $50 \mu \mathrm{l}$ of RNase-free water.

\section{Quantitative Reverse Transcription-Polymerase Chain Reaction}

Quantitative reverse transcription-polymerase chain reaction (qRT-PCR) was performed in a Chromo4 RealTime PCR Detection System (Bio-Rad Laboratories, Hercules, CA, USA) using TaqMan MicroRNA Reverse Transcription Kit and TaqMan Universal PCR Master Mix (Applied Biosystems, Tokyo, Japan). For the measurement of miRNA expression, we performed two-step qRT-PCR with specific primers for the indicated miRNAs (designed by Applied Biosystems) following the manufacturer's protocol. Each sample was run in triplicate. The level of miRNA expression was calculated from a standard curve constructed by using small RNAs from CAPAN-1 cells. The expression levels of the indicated miRNAs were normalized to those of RNU6B. The accuracy and integrity of the PCR products were confirmed with an Agilent 2100 Bioanalyzer (Agilent Technologies Inc., Palo Alto, CA, USA).

\section{Statistical Analysis}

A data mining technique provided by the SAS Institute was used to split gene expression in high- and low-level groups on the basis of a platform that recursively partitions data according to a relationship between the $X$ and $Y$ values, creating a tree of partitions (recursive descent partition analysis). ${ }^{26}$ By searching all possible cuts, it finds a set of cut points of $X$ values (gene expression) that best predict the $Y$ value (survival time). These data splits are done, recursively forming a tree of decision rules until the desired fit is reached; the most significant split is determined by the largest likelihood ratio chi-square statistic. In either case, the split is chosen to maximize the difference in the responses between the two branches of the split. 
Categorical variables were compared by a chi-square test (Fisher's exact probability test). Survival curves were constructed using the Kaplan-Meier product-limit method and compared by the log rank test. To evaluate the independent prognostic factors associated with patient survival (with or without gemcitabine treatment), multivariate Cox proportional hazard regression analysis was used, with miR-142-5p expression, miR-34a expression, $\mathrm{pN}$ status, and/or residual tumor status ( $\mathrm{R}$ factor) as covariates. Statistical significance was defined as a $P$ value of $<0.05$. All statistical analyses were performed by JMP 7.01 software (SAS Institute, Cary, NC, USA).

\section{RESULTS}

\section{Establishment of Gemcitabine-Resistant Pancreatic Cancer Cells}

Gemcitabine-resistant SUIT-2 and CAPAN-1 cells were generated by exposure to gradually increasing concentrations of gemcitabine. The final concentration of gemcitabine was $200 \mathrm{nM}$ for SUIT-2 cells and $1 \mu \mathrm{M}$ for CAPAN-1 cells. The viability of the parental SUIT-2 cells significantly decreased to less than $20 \%$ after treatment with $10 \mathrm{nM}$ gemcitabine, while the viability of gemcitabine-resistant SUIT-2 cells remained unchanged after treatment with $10 \mathrm{nM}$ gemcitabine. The viability of the parental CAPAN-1 cells significantly decreased to less than $10 \%$ after treatment with $1 \mu \mathrm{M}$ gemcitabine, while the viability of gemcitabine-resistant CAPAN-1 cells remained unchanged after treatment with $1 \mu \mathrm{M}$ gemcitabine.

\section{miRNA Differentially Expressed between Parent and Gemcitabine-Resistant Cells}

We used the TaqMan miRNA array to obtain the 365 miRNA expression profiles from the parent and gemcitabine-resistant pancreatic cancer cell lines. The results show that the expression of 10 miRNAs was more than 2-fold higher in both SUIT-2 and CAPAN-1 gemcitabine-resistant cells compared with the parental cells. We also identified 14 miRNAs in both SUIT-2 and CAPAN-1 gemcitabineresistant cells that were downregulated to less than $30 \%$ of that in the parental cells.

To confirm our TaqMan miRNA array data, we subjected 11 of the differentially expressed miRNAs (5 upregulated miRNAs: miR-9, miR-27a, miR-424, miR449b, and miR-34a; 6 downregulated miRNAs: miR-152, miR-181c, miR-518b, miR-125a, miR-142-5p, and miR204), which were selected on the basis of expression levels and fold changes as promising candidate of clinical markers, to triplicate qRT-PCR analysis, and obtained consistent results (data not shown).
Analysis of miRNA Expression in Relation to Survival Time of Patients with Pancreatic Cancer after Curative Resection

Conventional prognostic factors, such as pT category, $\mathrm{pN}$ category, $\mathrm{R}$ factor, positive vessel invasion, and adjuvant therapies, including 5-fluorouracil or gemcitabine, reached significance for overall survival (Table 1 ). We also found that the patients treated with gemcitabine had a better prognosis than those without, although the difference was not significant $(P=0.058)$.

Next, to investigate the correlation between gemcitabine response and miRNA expression identified by miRNA expression profiling, patients with resected pancreatic cancer were divided into two groups: patients who were treated with gemcitabine and patients who were not. Within each group, we investigated the correlation between the expression levels of the 11 miRNAs identified by miRNA expression profiling and the prognosis of patients with resected pancreatic cancer. miRNA expression was also divided into high- and low-level groups by recursive descent partition analysis, as described by Hoffmann et al. ${ }^{26}$

First, we investigated the correlation between prognosis and the six downregulated miRNAs: miR-152, miR-181c, miR-518b, miR-125a, miR-142-5p, and miR-204. For the gemcitabine group, the high miR-142-5p and miR-204 patients had a significantly longer survival time than the low miR-142-5p and miR-204 patients (Figs. 1 and 2, $P=0.0077$ for miR-142-5p; $P=0.0054$ for $\mathrm{miR}-204)$. The median survival time was 45 months and 33 months, respectively, for the high miR-142-5p and high miR-204 patients, and 16.3 months in both the low miR-142-5p and low miR-204 patients (Table 2). We also found that the high miR-125a patients had significantly longer survival times than the low miR-125a patients when the Wilcoxon test was used $(P=0.035)$, although the difference was not statistically significant when the log rank test was used (Table 2, $P=0.085$ ). However, for the nongemcitabine group, there were no differences in the survival times between the high and low miR-142-5p, miR-204, or miR125a patients (Table 2). Analysis of both the gemcitabine and nongemcitabine groups did not revealed any significant differences in survival time between the high and low miR152, miR-181c, and miR-518b patients (data not shown).

Next, we investigated the correlation between prognosis and the six upregulated miRNAs: miR-9, miR-27a, miR424, miR-449b, and miR-34a. For the nongemcitabine group, the high miR-34a patients had a significantly longer survival times than the low miR-34a patients (Table 2, $P=0.012$ ), while there were no significant differences in survival time between the high and low miR-34a patients in the gemcitabine group (Table $2, P=0.175$ ). The median 
FIG. 1 Correlation between the expression levels of miR$142-5 p$ identified by miRNA expression profiling and the prognosis of patients with resected pancreatic cancer in the gemcitabine and nongemcitabine groups. The levels of miRNA expression were normalized against RNU6B. High miR-142-5p expression was significantly associated with longer survival times in the gemcitabine group $(P=0.0077)$, but not in the nongemcitabine group $(P=0.48)$

FIG. 2 The correlation between the expression levels of miR-204 identified by miRNA expression profiling and the prognosis of patients with resected pancreatic cancer in the gemcitabine and nongemcitabine groups. The levels of miRNA expression were normalized against RNU6B. High miR-204 expression was significantly associated with longer survival times in the gemcitabine group $(P=0.0054)$, but not in the nongemcitabine group $(P=0.15)$
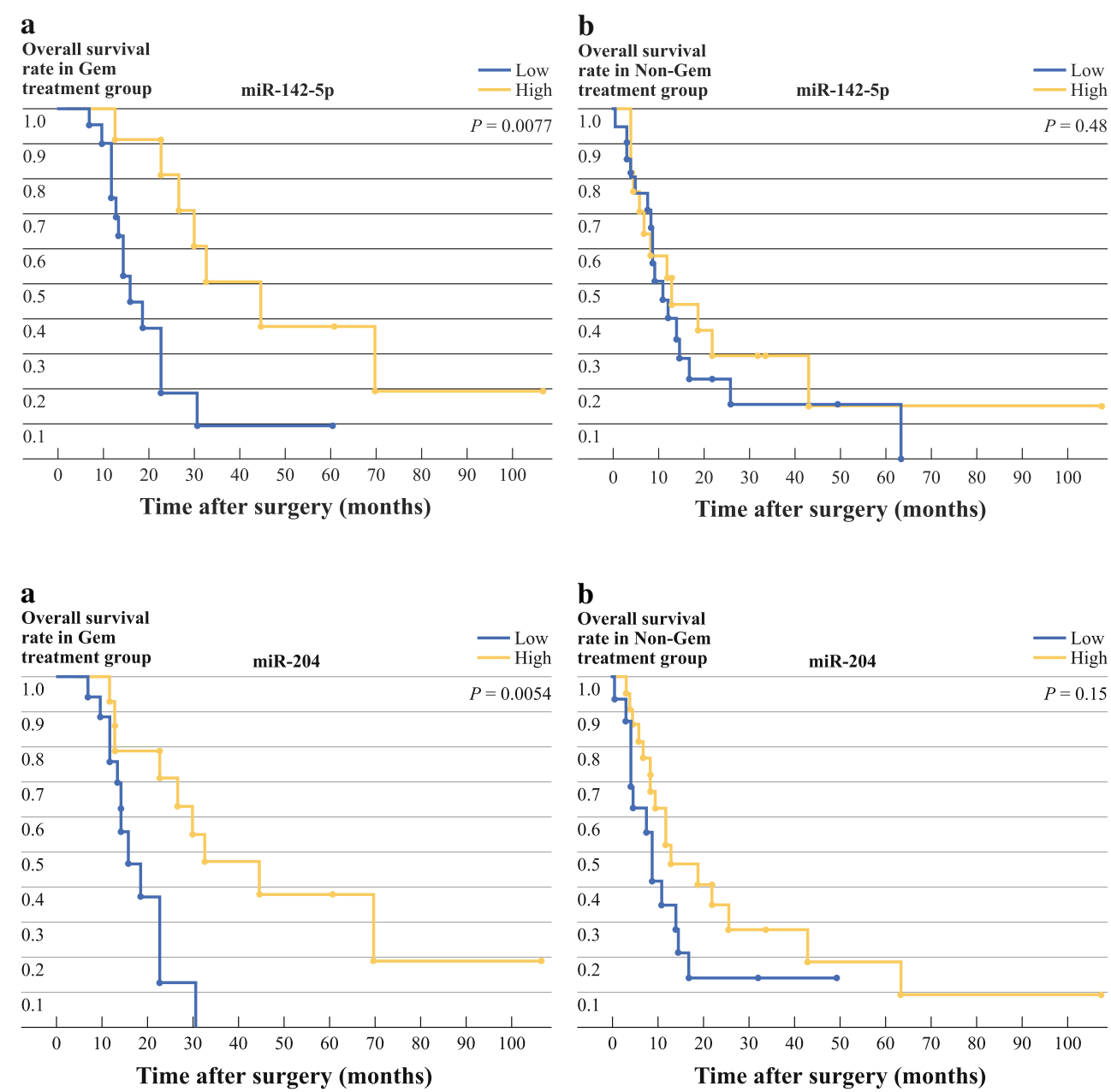

TABLE 2 Correlation between median overall survival and microRNA expression in patients with resected pancreatic cancer

\begin{tabular}{llll}
\hline Factor & \multicolumn{2}{l}{ Overall survival $(95 \%$ CI $)$} & $P$ value \\
\cline { 2 - 3 } & High & Low & \\
\hline miR-142-5p & & $16.3(12-23)$ & 0.0077 \\
$\quad$ Gem group & $45(23-)$ & $11(7.7-14.7)$ & 0.4880 \\
Non-Gem group & $13.07(4.7-43)$ & & \\
miR-204 & & $16.3(12-23)$ & 0.0054 \\
Gem group & $33(13.27-)$ & $9(4-14.7)$ & 0.1527 \\
Non-Gem group & $13.07(8.47-26)$ & & \\
miR-125a & & $14.57(12-31)$ & 0.0854 \\
Gem group & $30.17(19-)$ & $14(3-)$ & 0.8990 \\
Non-Gem group & $12(8.47-16.93)$ & & 0.1754 \\
miR-34a & & $16.3(12-)$ & 0.0127 \\
Gem group & $27(20.87-47.9)$ & $8.5(3-12)$ & \\
Non-Gem group & $16.9(9-24.4)$ & &
\end{tabular}

CI confidence interval, Gem gemcitabine

survival time was 16.9 months in the high miR-34a patients and 8.5 months in the low miR-34a patients in the nongemcitabine group (Table 2). We also found no significant differences in survival between the high and low miR-9, miR-27a, miR-424, and miR-449b patients in the gemcitabine and nongemcitabine groups (data not shown).

Multivariate Analysis of miR-142-5p and miR-34a Expression in Relation to Survival Time of Patients with or without Gemcitabine Treatment after Curative Resection

In three miRNAs identified as potential prognostic markers in the gemcitabine group, miR-142-5p was the best candidate and was the most specific for the gemcitabine group compared with the nongemcitabine group. Therefore, we focused on miR-142-5p as the most promising predictive marker for gemcitabine. Also, univariate analysis of the gemcitabine group showed that the only significant clinical factors were $\mathrm{pN}$ status ( $\mathrm{pN} 1$ vs. $\mathrm{pN}$ ), and R factor (R1 vs. R0). Multivariate survival analysis, based on the Cox proportional hazard model, was performed by using miR-142$5 \mathrm{p}$ expression (high versus low), $\mathrm{pN}$ status, and $\mathrm{R}$ factor. Overall survival time was significantly dependent on miR142-5p expression (Table $3, P=0.034$ ), but not on $\mathrm{pN}$ 
TABLE 3 Multivariate survival analysis (Cox regression model) of clinical prognostic factors and miR-142-5p expression in patients with gemcitabine treatment

\begin{tabular}{llll}
\hline Factor & Relative risk & $95 \%$ CI & $P$ value \\
\hline miR-142-5p & 3.109 & $1.087-10.01$ & 0.0340 \\
pN status & 2.055 & $0.646-9.219$ & 0.2395 \\
R factor & 2.197 & $0.823-5.904$ & 0.1146 \\
\hline
\end{tabular}

CI confidence interval

status $(P=0.24)$ or $\mathrm{R}$ factor $(P=0.11)$. In the gemcitabine group, miR-142-5p expression was an independent prognostic marker for pancreatic cancer patients, with a relative risk of 3.109 (Table 3).

Univariate analysis of the nongemcitabine group showed that the only significant clinical factor was $\mathrm{pN}$ status. Therefore, multivariate survival analysis was performed by using miR-34a expression and $\mathrm{pN}$ status. The results show that the overall survival time was significantly dependent on both miR-34a expression and $\mathrm{pN}$ status (Table $4, P=0.0103$ for miR-34a; $P=0.0035$ for $\mathrm{pN}$ status). In the nongemcitabine group, miR-34a expression was an independent prognostic marker for pancreatic cancer patients with a relative risk of 2.920 (Table 4).

\section{DISCUSSION}

The present miRNA profiling study using two gemcitabine-resistant pancreatic cancer cell lines and their parent cell lines identified 24 miRNAs candidates, which were upor downregulated in gemcitabine resistant cells. The present results also showed that high miR-142-5p and miR-204 patients had statistically significantly longer survival times than the low miR-142-5p and miR-204 patients in the gemcitabine group, but not in nongemcitabine group, although further examination is needed because the number of patients in the nongemcitabine group is too small to conclude that there were no differences in survival between high and low expression patients in the nongemcitabine group. Liu et al. reported that miR-142-5p was repressed in human lung cancer, and the transfection of miR-142-5p significantly repressed lung cancer cell growth. ${ }^{27}$ miR-204 has been also reported to be downregulated in intrahepatic cholangiocarcinoma, and the level of miR-204 expression

TABLE 4 Multivariate survival analysis (Cox regression model) of clinical prognostic factors and miR-34a expression in patients without gemcitabine treatment

\begin{tabular}{llll}
\hline Factor & Relative risk & $95 \%$ CI & $P$ value \\
\hline miR-34a & 2.920 & $1.303-6.295$ & 0.0103 \\
pN status & 2.957 & $1.410-6.812$ & 0.0035 \\
\hline
\end{tabular}

CI confidence interval was inversely correlated with that of Bcl-2 expression, possibly leading to chemotherapeutic drug-triggered apoptosis. $^{28}$ Taken together, these data suggest that miR142-5p and miR-204 are promising predictive markers for chemotherapeutic responses in patients with resected pancreatic cancer.

In neuroblastomas, miR-34a was generally expressed at lower levels in unfavorable primary tumors, and the reintroduction of miR-34a results in a dramatic reduction in cell proliferation. ${ }^{29}$ miR-34a expression was decreased in 9 of $25(36 \%)$ colon cancers, and transient introduction of miR-34a suppressed the in vitro and in vivo growth of colon cancer. ${ }^{30}$ In non-small-cell lung cancer, the miR-34 family was downregulated in tumors compared with normal tissues, and low levels of miR-34a expression correlated with a high probability of relapse. ${ }^{31}$ These data suggest that miR-34a functions as a potential tumor suppressor. Our results show that miR-34a is a favorable prognostic marker in patients without gemcitabine treatment after resection. We also found no correlation between miR-34a expression and survival time in patients treated with gemcitabine, possibly suggesting that pancreatic cancers with low levels of miR-34a are more sensitive to gemcitabine treatment than those with high levels of miR$34 \mathrm{a}$, although larger studies are needed to confirm this.

In conclusion, miR-142-5p expression is correlated with survival time in patients treated with gemcitabine after surgical resection of pancreatic cancer, but not in patients without gemcitabine treatment. miR-142-5p would be a promising predictive marker for gemcitabine treatment in patients with resected pancreatic cancer, although further examination are needed to analyze the functional role of these microRNAs. In the present study, we used FFPE samples to measure the indicated miRNAs. Measurement of such miRNAs may be possible using plasma, serum, and pancreatic fluids, suggesting that these miRNAs may be useful in predicting the effects of chemotherapy for unresectable pancreatic cancer and of neoadjuvant chemotherapy for resectable pancreatic cancer, where it is difficult to obtain tissues samples without the use of invasive procedures.

ACKNOWLEDGMENT Supported in part by a Grant-in-Aid from the Ministry of Education, Culture, Sports, Science and Technology of Japan, and a grant from the Kato Memorial Bioscience Foundation. We are grateful to Emiko Manabe, and Miyuki Omori (Department of Surgery and Oncology, Kyushu University) for skillful technical assistance.

\section{CONFLICT OF INTEREST None.}

OPEN ACCESS This article is distributed under the terms of the Creative Commons Attribution Noncommercial License which permits any noncommercial use, distribution, and reproduction in any medium, provided the original author(s) and source are credited. 


\section{REFERENCES}

1. Jemal A, Siegel R, Ward E, Murray T, Xu J, Thun MJ. Cancer statistics, 2007. CA Cancer J Clin. 2007;57:43-66.

2. Hirata K, Egawa S, Kimura Y, et al. Current status of surgery for pancreatic cancer. Dig Surg. 2007;24:137-47.

3. Hawes RH, Xiong Q, Waxman I, Chang KJ, Evans DB, Abbruzzese JL. A multispecialty approach to the diagnosis and management of pancreatic cancer. Am J Gastroenterol. 2000; 95:17-31.

4. Matsuno S, Egawa S, Fukuyama S, et al. Pancreatic Cancer Registry in Japan: 20 years of experience. Pancreas. 2004;28: 219-30.

5. Neoptolemos JP, Stocken DD, Friess H, et al. A randomized trial of chemoradiotherapy and chemotherapy after resection of pancreatic cancer. $N$ Engl J Med. 2004;350:1200-10.

6. Oettle H, Post S, Neuhaus P, et al. Adjuvant chemotherapy with gemcitabine vs observation in patients undergoing curative-intent resection of pancreatic cancer: a randomized controlled trial. JAMA. 2007;297:267-77.

7. Heinemann V, Hertel LW, Grindey GB, Plunkett W. Comparison of the cellular pharmacokinetics and toxicity of $2^{\prime}, 2^{\prime}$-difluorodeoxycytidine and 1-beta-D-arabinofuranosylcytosine. Cancer Res. 1988;48:4024-31.

8. Burris HA III, Moore MJ, Andersen J, et al. Improvements in survival and clinical benefit with gemcitabine as first-line therapy for patients with advanced pancreas cancer: a randomized trial. $J$ Clin Oncol. 1997;15:2403-13.

9. Moore MJ, Goldstein D, Hamm J, et al. Erlotinib plus gemcitabine compared with gemcitabine alone in patients with advanced pancreatic cancer: a phase III trial of the National Cancer Institute of Canada Clinical Trials Group. J Clin Oncol. 2007;25:1960-6.

10. Van Cutsem E, Vervenne WL, Bennouna J, et al. Phase III trial of bevacizumab in combination with gemcitabine and erlotinib in patients with metastatic pancreatic cancer. J Clin Oncol. 2009;27: 2231-7.

11. Farrell JJ, Elsaleh H, Garcia M, et al. Human equilibrative nucleoside transporter 1 levels predict response to gemcitabine in patients with pancreatic cancer. Gastroenterology. 2009;136: 187-95.

12. Nakano Y, Tanno S, Koizumi K, et al. Gemcitabine chemoresistance and molecular markers associated with gemcitabine transport and metabolism in human pancreatic cancer cells. $\mathrm{Br} \mathrm{J}$ Cancer. 2007;96:457-63.

13. Wolff AC, Hammond ME, Schwartz JN, et al. American Society of Clinical Oncology/College of American Pathologists guideline recommendations for human epidermal growth factor receptor 2 testing in breast cancer. J Clin Oncol. 2007;25:118-45.

14. Lagos-Quintana M, Rauhut R, Lendeckel W, Tuschl T. Identification of novel genes coding for small expressed RNAs. Science. 2001;294:853-8.

15. Lau NC, Lim LP, Weinstein EG, Bartel DP. An abundant class of tiny RNAs with probable regulatory roles in Caenorhabditis elegans. Science. 2001;294:858-62.

16. Li J, Smyth P, Flavin R, et al. Comparison of miRNA expression patterns using total RNA extracted from matched samples of formalin-fixed paraffin-embedded (FFPE) cells and snap frozen cells. BMC Biotechnol. 2007;7:36.
17. Xi Y, Nakajima G, Gavin E, et al. Systematic analysis of microRNA expression of RNA extracted from fresh frozen and formalin-fixed paraffin-embedded samples. RNA. 2007;13: $1668-74$.

18. Lebanony D, Benjamin H, Gilad S, et al. Diagnostic assay based on hsa-miR-205 expression distinguishes squamous from nonsquamous non-small-cell lung carcinoma. J Clin Oncol. 2009;27: 2030-7.

19. Bloomston M, Frankel WL, Petrocca F, et al. MicroRNA expression patterns to differentiate pancreatic adenocarcinoma from normal pancreas and chronic pancreatitis. JAMA. 2007;297: 1901-8.

20. Xie Y, Todd NW, Liu Z, et al. Altered miRNA expression in sputum for diagnosis of non-small cell lung cancer. Lung Cancer. 2010;67:170-6.

21. Greither T, Grochola L, Udelnow A, Lautenschlager C, Wurl P, Taubert H. Elevated expression of microRNAs 155, 203, 210 and 222 in pancreatic tumours associates with poorer survival. Int $J$ Cancer. 2010;126(1):73-80.

22. Dillhoff M, Liu J, Frankel W, Croce C, Bloomston M. MicroRNA-21 is overexpressed in pancreatic cancer and a potential predictor of survival. J Gastrointest Surg. 2008;12:2171-6.

23. Ohuchida K, Mizumoto K, Murakami M, et al. Radiation to stromal fibroblasts increases invasiveness of pancreatic cancer cells through tumor-stromal interactions. Cancer Res. 2004;64: 3215-22.

24. Hamilton R, Gray C, Belanger SA, et al. Cardiac risk assessment before the use of stimulant medications in children and youth: a joint position statement by the Canadian Paediatric Society, the Canadian Cardiovascular Society and the Canadian Academy of Child and Adolescent Psychiatry. J Can Acad Child Adolesc Psychiatry. 2009;18:349-55.

25. Sobin C, Gutierrez M, Alterio H. Polymorphisms of delta-aminolevulinic acid dehydratase (ALAD) and peptide transporter 2 (PEPT2) genes in children with low-level lead exposure. $\mathrm{Neu}$ rotoxicology. 2009;30:881-7.

26. Hoffmann AC, Mori R, Vallbohmer D, et al. High expression of HIF1a is a predictor of clinical outcome in patients with pancreatic ductal adenocarcinomas and correlated to PDGFA, VEGF, and bFGF. Neoplasia. 2008;10:674-9.

27. Liu X, Sempere LF, Galimberti F, et al. Uncovering growthsuppressive microRNAs in lung cancer. Clin Cancer Res. 2009; 15:1177-83.

28. Chen L, Yan HX, Yang W, et al. The role of microRNA expression pattern in human intrahepatic cholangiocarcinoma. J Hepatol. 2009;50:358-69.

29. Welch C, Chen Y, Stallings RL. MicroRNA-34a functions as a potential tumor suppressor by inducing apoptosis in neuroblastoma cells. Oncogene. 2007;26:5017-22.

30. Tazawa H, Tsuchiya N, Izumiya M, Nakagama H. Tumor-suppressive miR-34a induces senescence-like growth arrest through modulation of the E2F pathway in human colon cancer cells. Proc Natl Acad Sci USA. 2007;104:15472-7.

31. Gallardo E, Navarro A, Vinolas N, et al. miR-34a as a prognostic marker of relapse in surgically resected non-small-cell lung cancer. Carcinogenesis. 2009;30:1903-9. 